\title{
Light Absorption by Metals with Porous Surface Layer Formed by Oxidization-Reduction Treatment
}

\author{
Masanori Suzuki*1, Toshifumi Yamamoto*2, Yohei Katayama*2, \\ Soichiro Kuwata*2 and Toshihiro Tanaka \\ Division of Materials and Manufacturing Science, Graduate School of Engineering, Osaka University, Suita 565-0871, Japan
}

Metals with fine surface asperity show higher efficiency of sunlight absorption than those with flat surfaces. In the present study, ultraviolet-visible-infrared light absorption by metals with porous surface layers was examined. A three-dimensionally interconnected porous structure was formed on the surface of copper and iron substrates by oxidizing and then reducing. Pore size and layer thickness were dependent on oxidization and reduction temperatures and times. It was found that metals with porous surface layers exhibited higher efficiencies of light absorption over a wide wavelength range than those with mirror-polished surfaces. High light absorption efficiencies were obtained for metal substrates with fine porous structures. In particular, a very fine porous structure was formed on the surface of iron substrate by oxidizing and reducing it at low temperatures, and its light absorption reached $75-80 \%$ over a wide wavelength range corresponding to ultraviolet-visibleinfrared light. This light absorption index is much higher than light absorption by conventional metal substrates with flat surface. [doi:10.2320/matertrans.M2012139]

(Received April 10, 2012; Accepted June 4, 2012; Published August 25, 2012)

Keywords: light absorption, porous structure, metal surface, oxidization, reduction

\section{Introduction}

Sunlight consists of ultraviolet-visible-infrared light, and these wavelengths could potentially provide a very high amount of energy. For highly efficient use of solar energy as an important heat supply, modification of surface structure has been performed on metallic materials. It has been found that metals with fine surface asperity exhibit higher efficiency of light absorption than those with flat surfaces as a result of multiple reflection of incoming light and multiple light absorption on the metal surface. ${ }^{1,2)}$ For instance, nano-scale surface asperity can be prepared on tungsten surfaces through application of plasma radiation. ${ }^{3)}$ The resulting concavoconvex metal surface provides high infrared light absorption, the energy of which could be exploited as thermal energy. However, it is difficult to apply conventional methods such as plasma radiation in preparing surface asperity on wide areas of metal plate. Therefore, a simple, low energy, and convenient method is required to make fine and homogeneous asperity structures on large metal surfaces.

As a simple and convenient method to prepare surface asperity on metal surfaces, the present study focuses on the formation of porous layers on metal surfaces by oxidizationreduction treatment. Hayes et al. ${ }^{4-6)}$ investigated the morphology of metals obtained by the reduction of metal oxides from the viewpoint of extractive metallurgy, and revealed that porous structures are formed in metallic materials during the reduction of metal oxides. In comparison, we previously attempted to prepare a porous layer on a metal surface by oxidization-reduction treatment, and observed unusual wetting of liquid metals on the porous metal substrate due to capillary phenomenon. ${ }^{7-9)}$ Our previous studies revealed that this porous structure was homogeneously formed on the metal surface, and was three-dimensionally interconnected.

\footnotetext{
${ }^{* 1}$ Corresponding author, E-mail: suzuki@mat.eng.osaka-u.ac.jp

${ }^{* 2}$ Graduate Student, Osaka University
}

The pore size was dependent on oxidizing and reducing temperature and time conditions. Since porous layers with nano-sized pores can be obtained on metal surfaces, reduced metal with a porous layer is expected to exhibit high efficiency of light absorption.

In the present study, we examine ultraviolet-visibleinfrared light absorption of metals with porous surface layers obtained by an oxidization-reduction process. Copper and iron substrates were selected based on our previous work in determining appropriate oxidizing and reducing conditions for formation of fine surface porous structures. ${ }^{7-9)}$ Various kinds of porous structure were prepared on the surface of copper and iron substrates under different oxidizing and reducing conditions. These surface structures were observed with scanning electron microscopy, and the effects of oxidizing and reducing conditions on pore size and thickness of the porous layer were investigated. Then, ultravioletvisible-infrared light absorption was examined to clarify the correlation between the obtained porous structure and the resulting light absorption of the metal substrates.

\section{Experimental Procedure}

\subsection{Preparation of porous layer on metal surface by oxidization-reduction treatment}

Purified copper $(99.994 \%)$ and iron $(99.5 \%)$ substrates (Nilaco Corp.), $20 \mathrm{~mm} \times 20 \mathrm{~mm} \times 1 \mathrm{~mm}$ in size, underwent oxidization-reduction treatments under the following conditions, to form porous layers on their surfaces.

Figure 1 shows a schematic diagram of the oxidization and reduction apparatus. Substrates were suspended in the center of the heated region, and kept in purified Ar atmosphere until the predetermined oxidizing or reducing temperature was achieved. The Ar gas (99.999\%) was purified by removal of water vapor and oxygen through silica gel, magnesium perchlorate and annealed magnesium shot, to prevent the metal substrate from being oxidized beyond that intended 


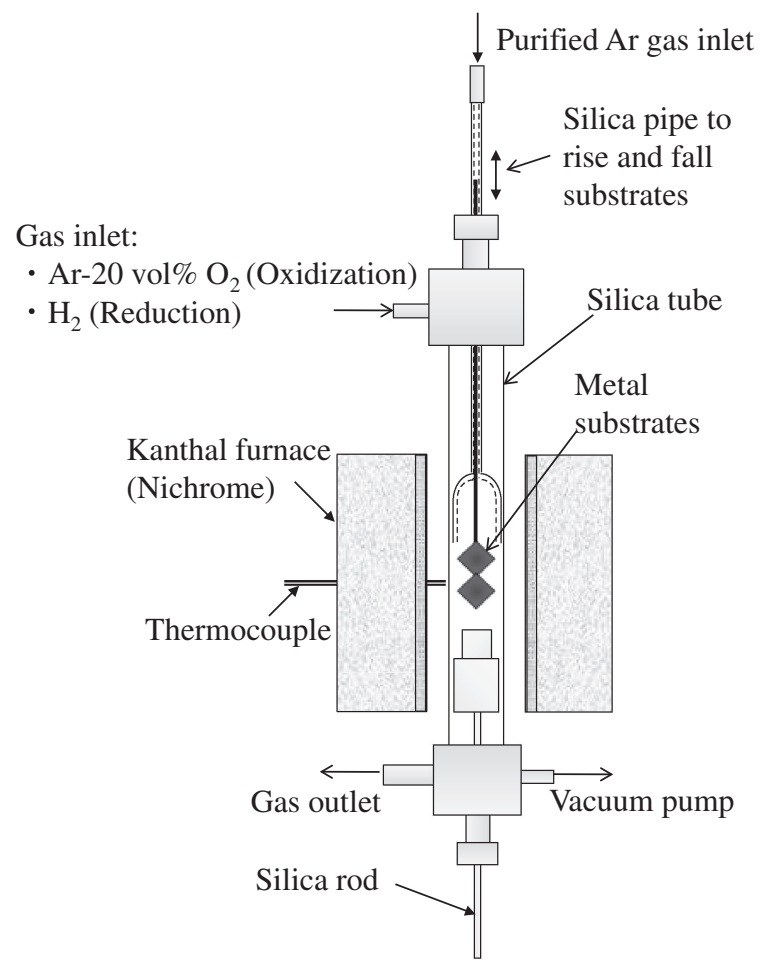

Fig. 1 Schematic diagram of metal substrate oxidization-reduction treatment apparatus.

from the oxidization-reduction treatment. Once the predetermined temperature was reached in the heated region, $\mathrm{Ar}-20$ vol\% $\mathrm{O}_{2}$ gas or purified $\mathrm{H}_{2}$ gas $(99.9999 \%)$ was introduced to conduct the oxidization-reduction treatment of the substrates. The oxidization-reduction treatment was terminated by reintroduction of the purified Ar gas, and by moving the substrate out of the heated region.

First, the copper and iron substrates were oxidized in $\mathrm{Ar}-$ $20 \mathrm{vol} \% \mathrm{O}_{2}$ atmosphere at a given temperature between 873 and $1073 \mathrm{~K}$ for $1 \mathrm{~h}$ maximum holding time. Then, to form the porous layer on the surface of each substrate, these oxidized substrates underwent a reducing process under purified $\mathrm{H}_{2}$ atmosphere. To prevent excessive coarsening of the obtained porous structure, the reducing holding temperature and time were carefully chosen. For copper substrates we selected temperatures between 473 and $673 \mathrm{~K}$, and for iron substrates between 773 and $923 \mathrm{~K}$, with $1 \mathrm{~h}$ as the maximum holding time for both substrates. Microstructures in the porous layers formed on the surface of the reduced copper and iron substrates were observed with a HITACHI TM-1000 scanning electron microscope (SEM), with an excitation voltage of $15 \mathrm{kV}$.

\subsection{Light absorption measurement by metals}

Ultraviolet-visible-infrared light absorption was examined for the samples with porous surface layers formed by the oxidization-reduction treatments, and the results were compared with those obtained for mirror polished metals to evaluate the effect of the porous structure on the metal surface. Light absorption by opaque solid material is generally evaluated by measuring reflectance of incoming light with different wavelengths, and by subtracting absolute reflectance ratio from unity. A SHIMADZU UV-3600 ultraviolet-visible-infrared spectrophotometer was used to measure relative spectral reflectivity in the wavelength range between 260 and $2200 \mathrm{~nm}$. $\mathrm{BaSO}_{4}$ powder was used as a standard reflectance material. Then, the absolute spectral absorption index for each metal substrate was determined by the following equation, using the absolute spectral reflectivity data of $\mathrm{BaSO}_{4}$ powder by Grum et al.: ${ }^{10)}$

$$
\begin{aligned}
& \text { Absolute spectral absorption index } \\
& \qquad \begin{array}{l}
= \\
\quad \times(\text { Absolative spectral reflectivity }) \\
\end{array}
\end{aligned}
$$

\section{Results and Discussion}

\subsection{Light absorption of copper substrates with porous layer formed by oxidization-reduction treatment}

Figure 2 shows surface and cross-sectional micrographs of copper substrates after oxidization for $1 \mathrm{~h}$ at $1073 \mathrm{~K}$ under $\mathrm{Ar}-20$ vol\% $\mathrm{O}_{2}$ atmosphere. A homogeneous cavernous structure was formed on the surface of the oxidized copper substrate, and the oxide phase corresponded to $\mathrm{CuO}$. The cross-sectional micrograph of the oxidized copper substrate revealed the presence of both $\mathrm{CuO}$ and $\mathrm{Cu}_{2} \mathrm{O}$ phases. The thickness of the $\mathrm{CuO}$ layer may have been dependent on the degree of the oxidization process, e.g., holding temperature and time.

Surface and cross-sectional micrographs of copper substrates reduced under $\mathrm{H}_{2}$ atmosphere for $1 \mathrm{~h}$ at different temperatures are shown in Fig. 3. Fine pores were observed in the cavernous surface structure, and the pore size increased as reducing temperature increased. Cross-sectional micrographs of the reduced copper substrates indicated that porosity decreased as the reduction holding temperature increased. In reducing the oxide phase on the substrates, the porous structure may have coarsened, thereby increasing pore size while decreasing porosity as holding temperature and time increased.

Light absorption measurements were carried out for the copper substrates oxidized at $1073 \mathrm{~K}$ and then reduced at different temperatures, the results of which are shown in Fig. 4. For comparison, light absorption was also measured for copper substrate with a mirror-polished surface. The copper substrates with porous layers formed by the oxidization-reduction treatments exhibited higher light absorption efficiencies in ultraviolet and visible light wavelengths than those with mirror-polished surfaces. In particular, light absorption of copper substrates with porous layers increased as reduction holding temperature decreased, because a finer porous structure was formed on the surface. Since porous structures on metal surface contribute to multiple reflection of incoming light and multiple light absorption on pore surface, light absorption is considered to be enhanced regardless of wavelengths.

However, the copper substrates exhibited very low infrared light absorption, too low to evaluate efficiency differences between the porous surface copper substrates and the mirrorpolished copper substrates for those wavelengths. To verify the enhancement of light absorption in infrared light wavelengths through the formation of porous metal surface 

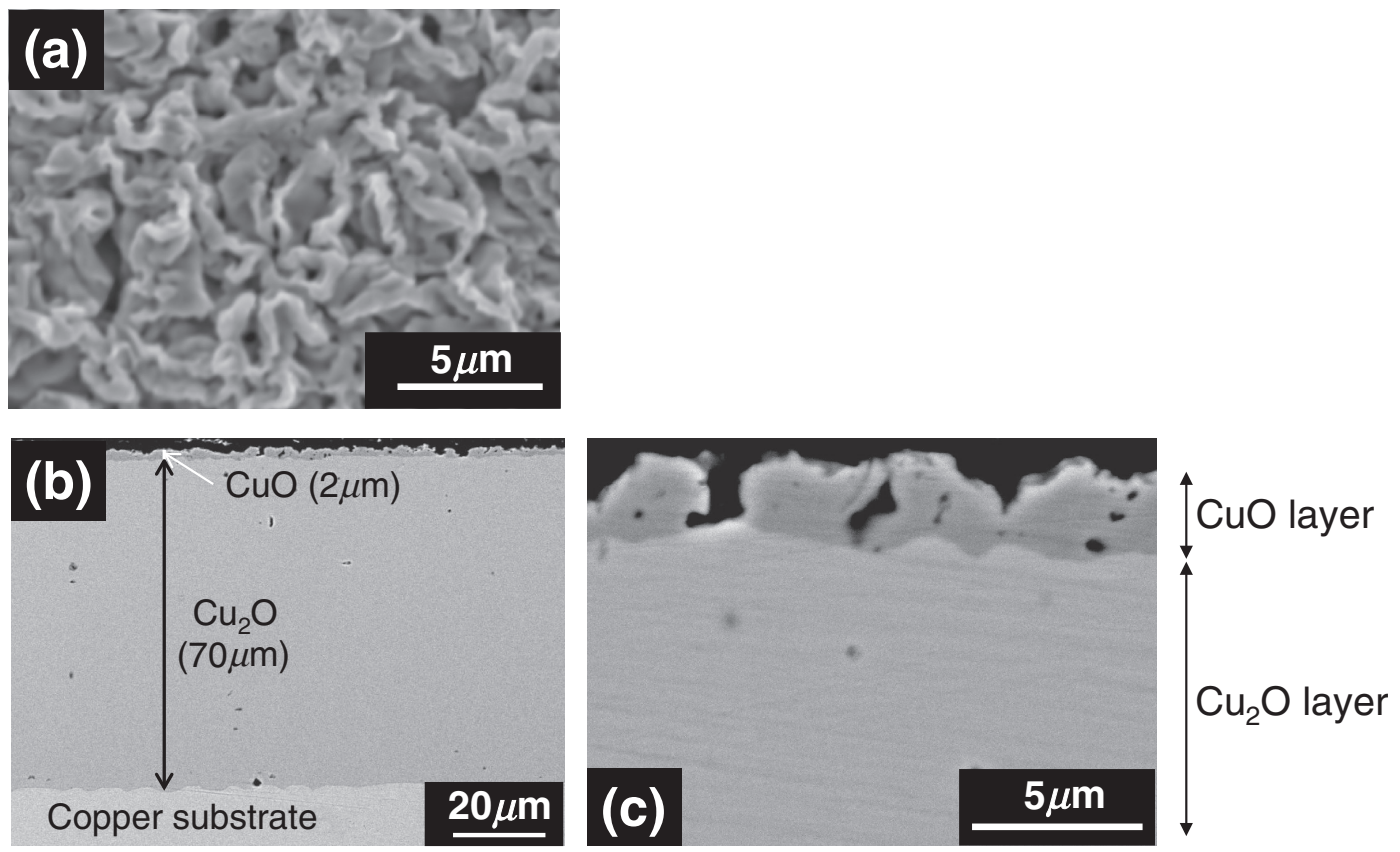

Fig. 2 Surface and cross-sectional micrographs of copper substrates after oxidizing at $1073 \mathrm{~K}, 1 \mathrm{~h}$ : (a) surface, (b) cross-section (low magnification), (c) cross-section (high magnification at surface).

Surface

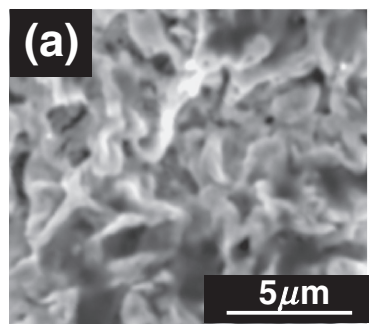

Cross-section

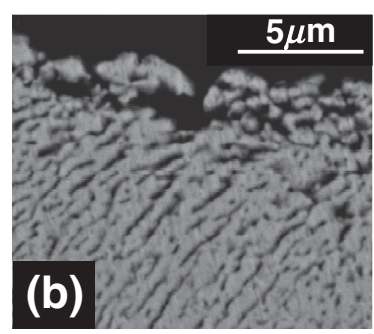

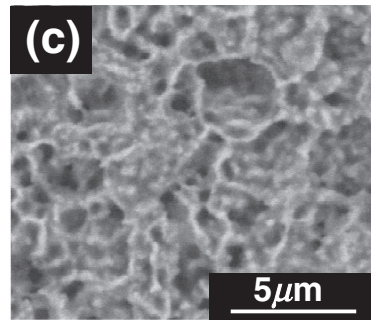

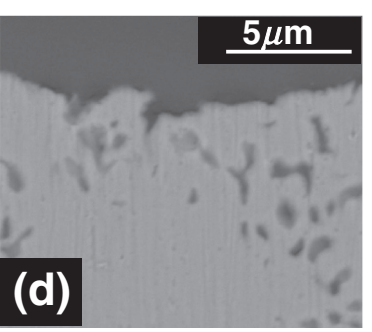

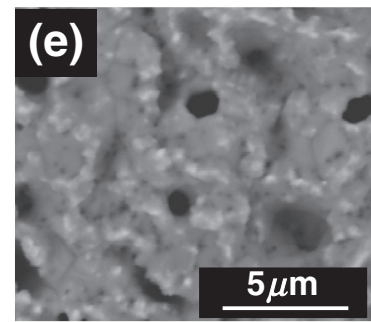

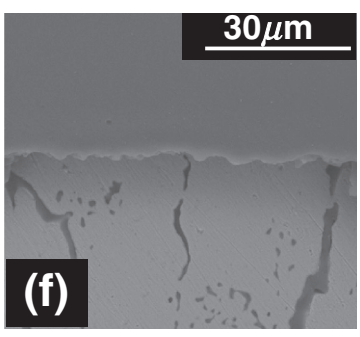

Fig. 3 Surface and cross-sectional micrographs of copper substrates after oxidizing for $1 \mathrm{~h}$ at $1073 \mathrm{~K}$ and reducing for $1 \mathrm{~h}$ at different temperatures, showing the effect of temperature in the reduction process: (a), (b) $473 \mathrm{~K}, 1 \mathrm{~h}$. (c), (d) $673 \mathrm{~K}, 1 \mathrm{~h}$. (e), (f) $873 \mathrm{~K}, 1 \mathrm{~h}$.

structures, it was necessary to select a metal substrate with intrinsic high efficiency of light absorption in infrared wavelengths.

\subsection{Light absorption of iron substrates with porous} layer formed by oxidization-reduction treatment

Figure 5 shows the measured light absorption efficiencies of mirror-polished iron and copper substrates over the ultraviolet-visible-infrared light wavelength range. The iron substrate exhibited high light absorption efficiency over a wider wavelength range than the copper substrate. Therefore, iron was confirmed to be a useful substrate material to verify the enhancement of infrared light absorption through formation of a porous surface structure by the present oxidization-reduction treatment.

Figure 6(a) shows a surface micrograph of the iron substrate after oxidization for $1 \mathrm{~h}$ at $1073 \mathrm{~K}$ under $\mathrm{Ar}-$ 20 vol\% $\mathrm{O}_{2}$ atmosphere; a cavernous structure was homogeneously formed on the oxidized iron substrate. Figures 6(b) and $6(\mathrm{c})$ show surface micrographs of previously oxidized iron substrates after reduction under $\mathrm{H}_{2}$ atmosphere for $1 \mathrm{~h}$ at 923 and $773 \mathrm{~K}$, respectively. Homogeneous porous structures were observed on the surface of the reduced substrates. The pore size for the substrate reduced at $923 \mathrm{~K}$ was measured at $1-2 \mu \mathrm{m}$. The surface morphology of the iron substrate after reduction at $773 \mathrm{~K}$ consisted of a fine porous structure and 


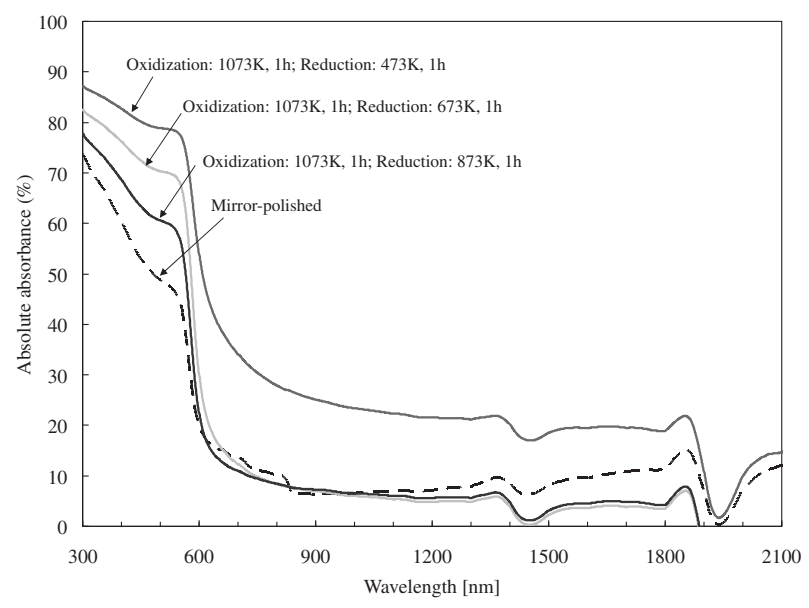

Fig. 4 Light absorption of copper substrates with porous layer formed by oxidizing for $1 \mathrm{~h}$ at $1073 \mathrm{~K}$ and reducing for $1 \mathrm{~h}$ at different temperatures.

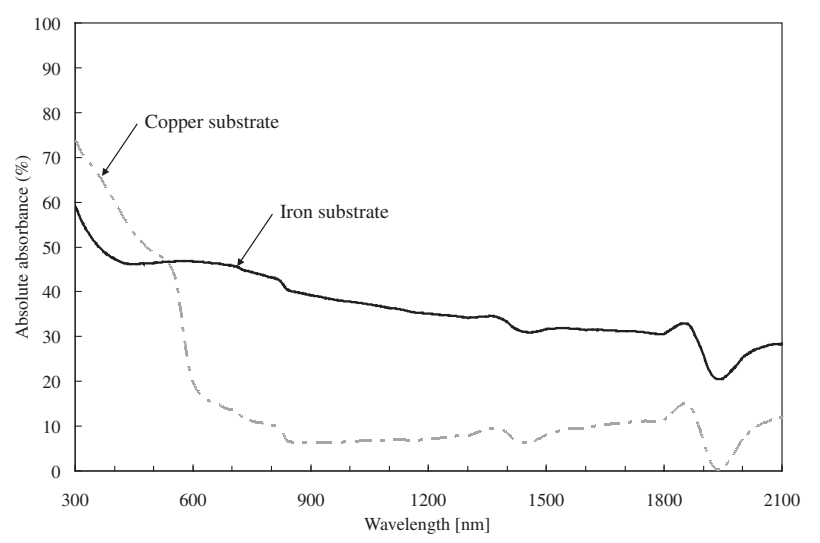

Fig. 5 Light absorption of iron and copper substrates with mirror-polished surface.
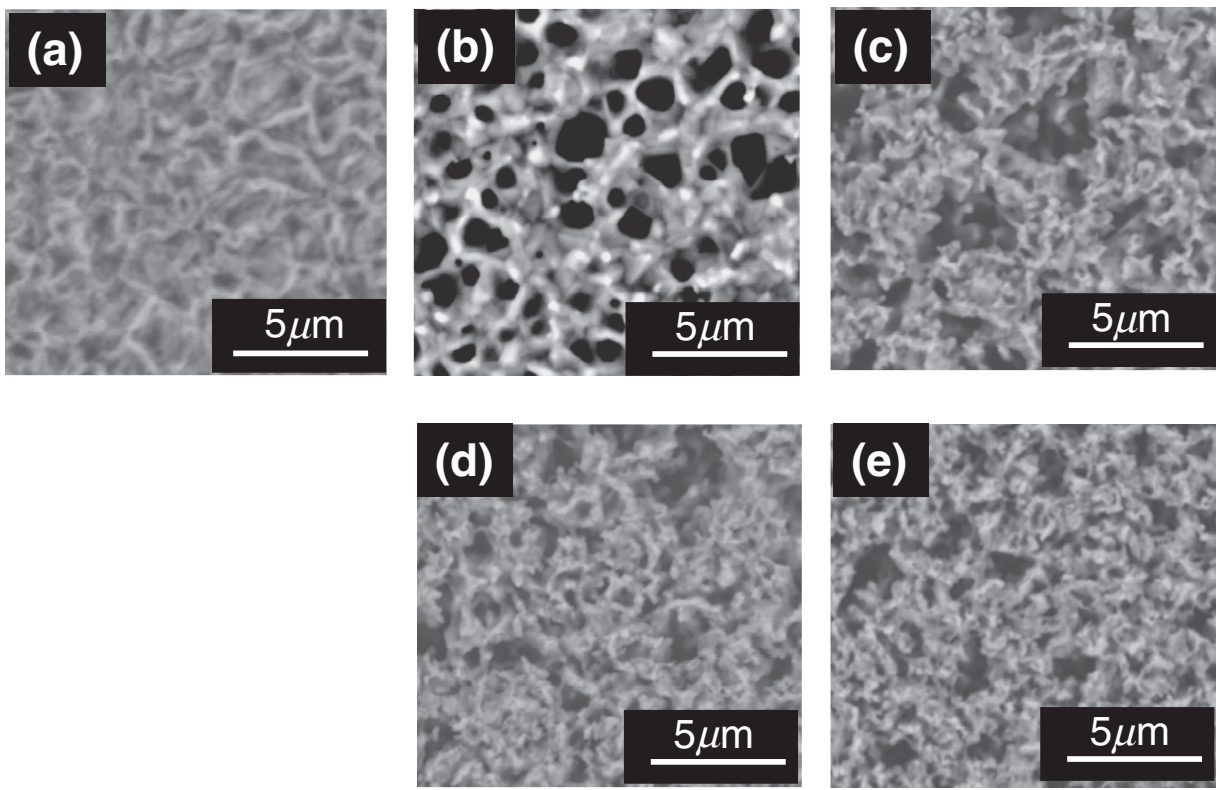

Fig. 6 Surface micrographs of iron substrates: (a) oxidized at $1073 \mathrm{~K}, 1 \mathrm{~h}$. (b) oxidized at $1073 \mathrm{~K}, 1 \mathrm{~h}$ and reduced at $923 \mathrm{~K}$, $1 \mathrm{~h}$. (c) oxidized at $1073 \mathrm{~K}, 1 \mathrm{~h}$ and reduced at $773 \mathrm{~K}, 1 \mathrm{~h}$. (d) Oxidized at $1073 \mathrm{~K}, 1 \mathrm{~h}$ and reduced at $773 \mathrm{~K}, 0.5 \mathrm{~h}$. (e) oxidized at $1073 \mathrm{~K}, 1 \mathrm{~h}$ and reduced at $773 \mathrm{~K}, 0.25 \mathrm{~h}$.

the remaining cavernous structure. The effect of reduction holding time on the surface of the iron substrates can be seen in Figs. 6(c), 6(d) and 6(e), which indicated that the increase in pore size with increasing reduction holding time was due to coarsening, similar to that observed for the oxidizationreduction treatments of copper substrates.

Figure 7 shows cross-sectional micrographs of iron substrates oxidized at $1073 \mathrm{~K}$ for $1 \mathrm{~h}$ under $\mathrm{Ar}-20 \mathrm{vol} \% \mathrm{O}_{2}$ atmosphere after reduction under $\mathrm{H}_{2}$ atmosphere at various temperatures and holding time. While porous layers were formed on the surfaces of these substrates as a result of the reduction of the oxidized layer, a remaining oxidized layer was also detected. It appeared that the oxidized iron layer formed at $1073 \mathrm{~K}, 1 \mathrm{~h}$, was too thick to be completely reduced. The thickness of the porous layer increased as reduction holding time increased. Vertical cracks accompanied the porous layer under reduction at $773 \mathrm{~K}$, these may have been formed by the decomposition of the wüstite phase in the oxidized substrate into iron and magnetite phases.

Figure 8 shows the results of light absorption measurements for iron substrates oxidized at $1073 \mathrm{~K}$ for $1 \mathrm{~h}$ and then reduced at different temperatures and holding times. It was observed that the iron substrates with porous surface layers formed by the oxidization-reduction treatment exhibited higher light absorption efficiencies than those with mirrorpolished surfaces over the entire measured range of ultraviolet-visible-infrared wavelengths. For example, the iron substrate oxidized at $1073 \mathrm{~K}$ for $1 \mathrm{~h}$ and reduced at $773 \mathrm{~K}$ for $0.25 \mathrm{~h}$ exhibited light absorption of $75-80 \%$ over the ultraviolet-visible-infrared wavelength range. This light absorption index is much higher than light absorption by conventional metal substrates with flat surface. Furthermore, it was also indicated that the light absorption of the reduced iron substrates increased as reducing temperature and holding 

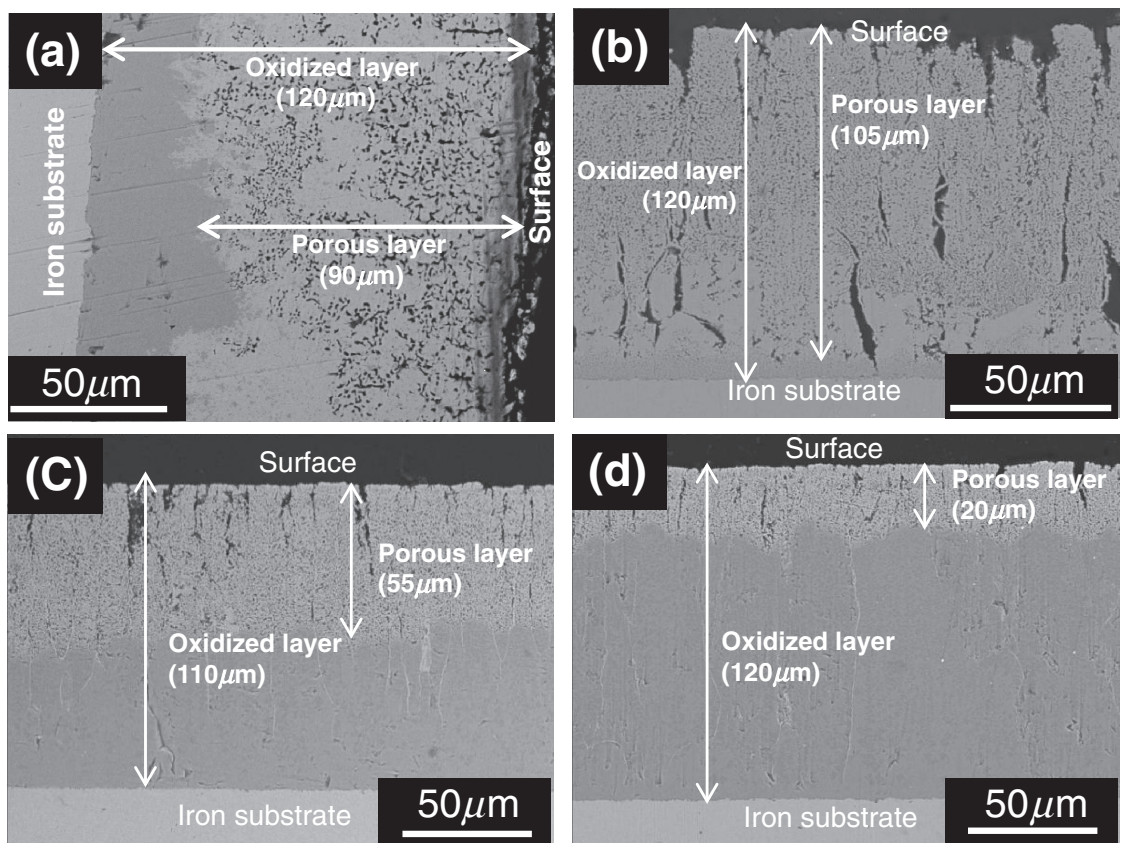

Fig. 7 Cross-sectional micrographs of iron substrates: (a) oxidized at $1073 \mathrm{~K}, 1 \mathrm{~h}$ and reduced at $923 \mathrm{~K}, 1 \mathrm{~h}$. (b) oxidized at $1073 \mathrm{~K}, 1 \mathrm{~h}$ and reduced at $773 \mathrm{~K}, 1 \mathrm{~h}$. (c) oxidized at $1073 \mathrm{~K}, 1 \mathrm{~h}$ and reduced at $773 \mathrm{~K}, 0.5 \mathrm{~h}$. (d) oxidized at $1073 \mathrm{~K}, 1 \mathrm{~h}$ and reduced at $773 \mathrm{~K}$, $0.25 \mathrm{~h}$.

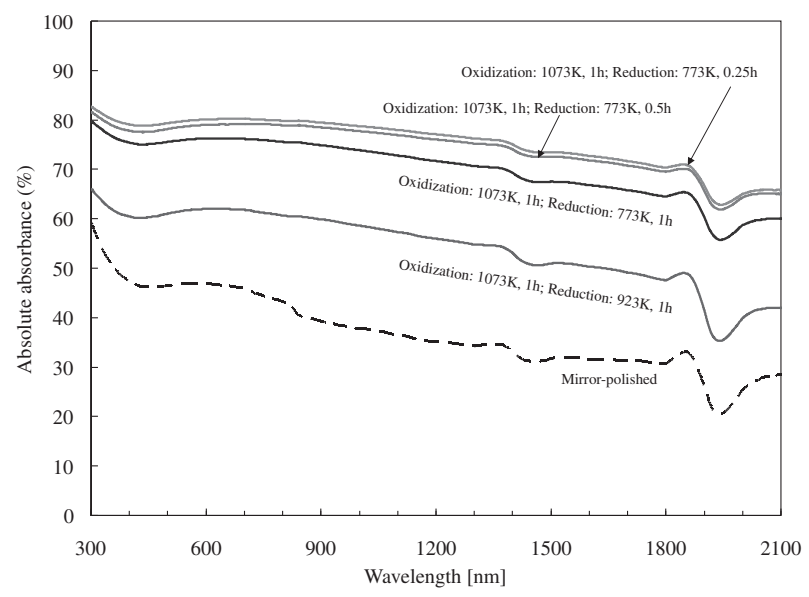

Fig. 8 Light absorption of iron substrates with porous layer formed by oxidizing at $1073 \mathrm{~K}, 1 \mathrm{~h}$ and reducing at different temperatures and times.

time decreased, showing that high efficiency of light absorption was achieved for iron substrates with fine porous surface structures.

The above experimental results revealed that the morphology of the porous layer on the surface of an iron substrate, e.g. pore size, could be controlled with temperature and time during the reduction process, and that the resulting light absorption of the iron substrates depended on the surface morphology formed by the oxidization-reduction treatment.

Subsequently, to obtain a thin porous layer of fine pore size on iron substrate, a low-temperature oxidization-reduction treatment was conducted. Figure 9 shows a surface micrograph of iron substrate after oxidization for $1 \mathrm{~h}$ at $873 \mathrm{~K}$ under $\mathrm{Ar}-20$ vol\% $\mathrm{O}_{2}$ atmosphere; a fine porous structure was homogeneously formed on surface. Figure 10 shows surface and cross-sectional micrographs of iron substrates reduced

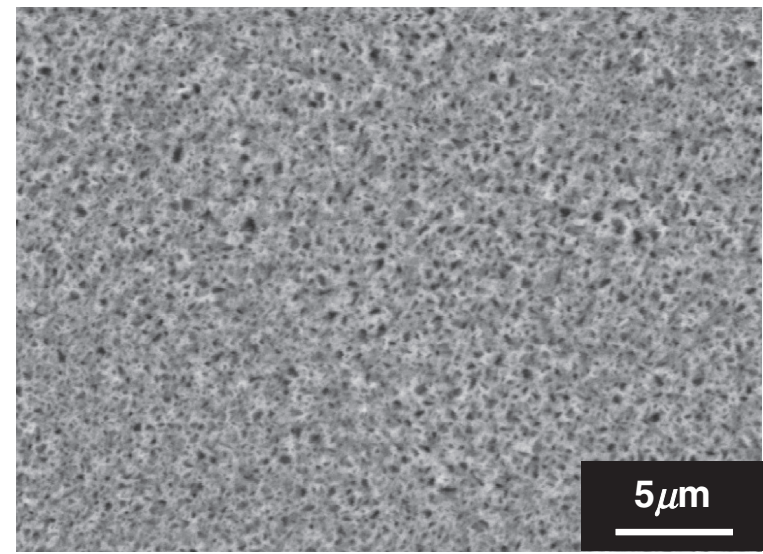

Fig. 9 Surface micrograph of iron substrate after oxidization at $873 \mathrm{~K}, 1 \mathrm{~h}$.

under $\mathrm{H}_{2}$ atmosphere at $773 \mathrm{~K}$ for different holding times. Fine porous structures were observed in the reduced iron substrates, and the pore size increased as holding time increased due to coarsening. Cross-sectional micrographs of the reduced iron substrates in Figs. 10(b), 10(d) and 10(f) indicated that no oxidized layer remained, and that the porous layer at the surface was much thinner than that formed on iron by oxidization at $1073 \mathrm{~K}$ and reduction at $773 \mathrm{~K}$, as shown in Fig. 7.

Figure 11 shows light absorption measurements for the iron substrates oxidized at $873 \mathrm{~K}$ for $1 \mathrm{~h}$ and then reduced at $773 \mathrm{~K}$ for different holding times. The oxidization-reduction treated iron substrates exhibited higher light absorption efficiencies than that of the mirror-polished substrate across the ultraviolet-visible-infrared light wavelengths. In particular, the iron substrate with a thin porous layer and fine porous structure, formed by oxidization at $873 \mathrm{~K}$ for $1 \mathrm{~h}$ and reduction at $773 \mathrm{~K}$ for $1 \mathrm{~h}$, exhibited light absorption of $75-$ 


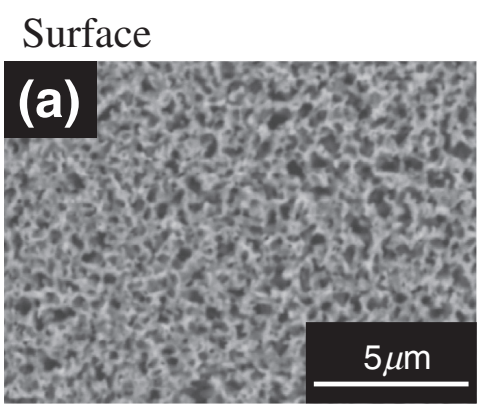

Cross-section

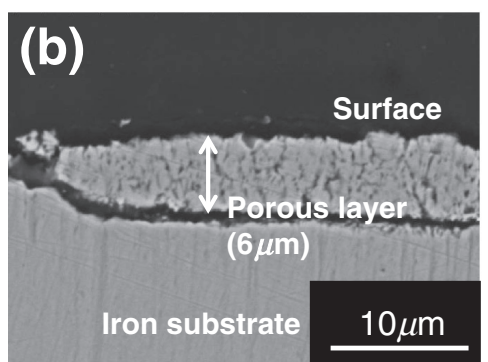

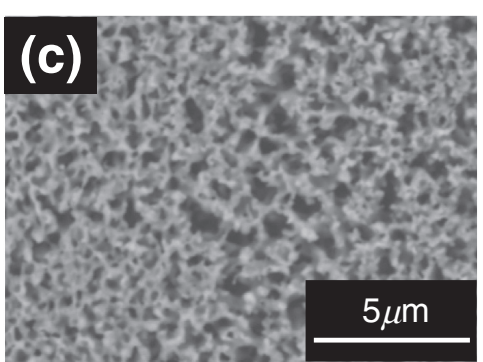
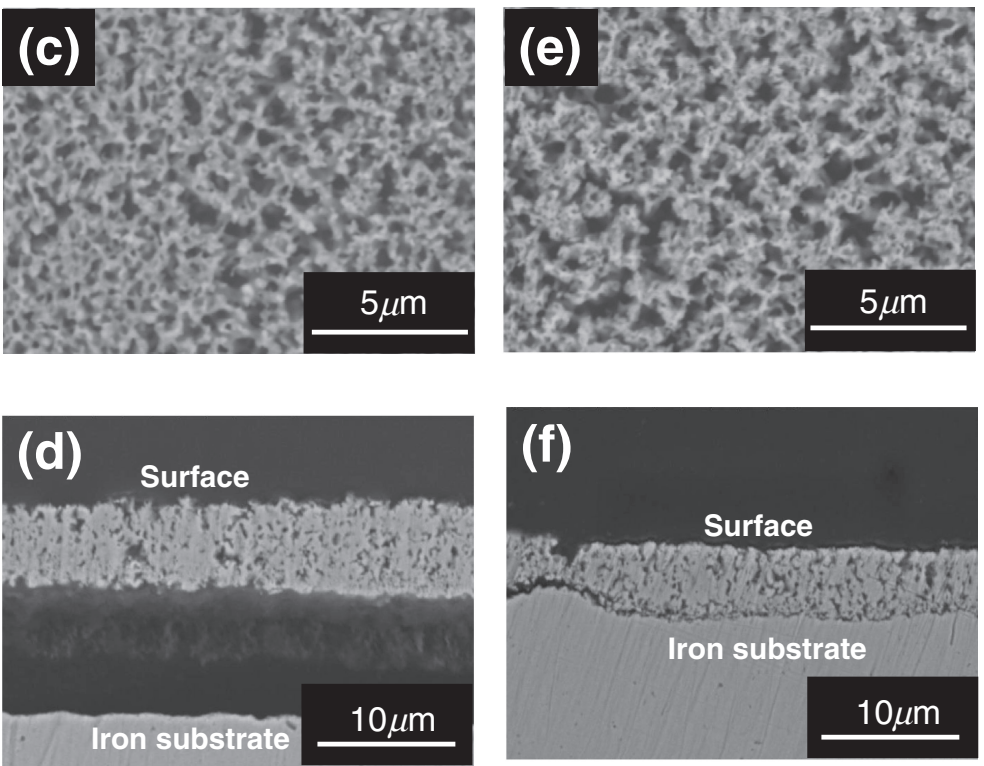

Fig. 10 Surface and cross-sectional micrographs of iron substrates after oxidizing at $873 \mathrm{~K}, 1 \mathrm{~h}$ and reducing at $773 \mathrm{~K}$ and different time, showing the effect of holding time in the reduction process: (a), (b) $773 \mathrm{~K}, 0.25 \mathrm{~h}$. (c), (d) $773 \mathrm{~K}, 0.5 \mathrm{~h}$. (e), (f) $773 \mathrm{~K}, 1 \mathrm{~h}$.

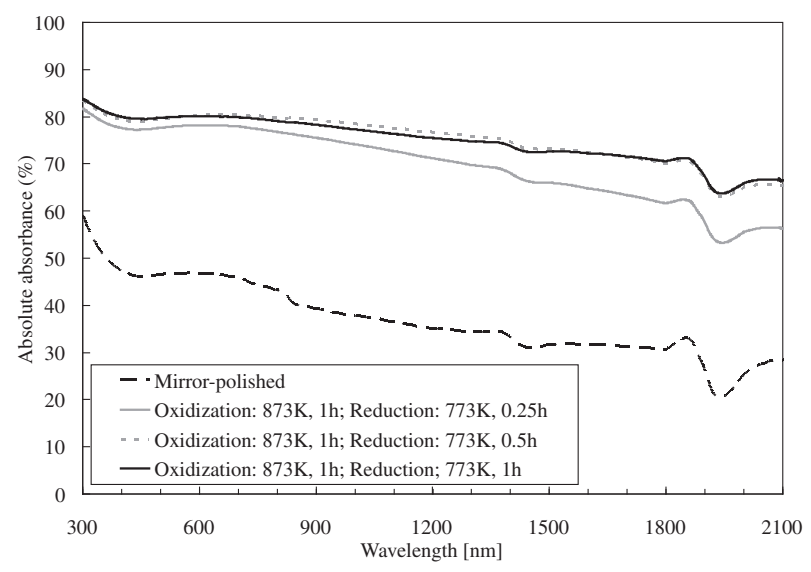

Fig. 11 Light absorption of iron substrates with porous layer formed by oxidizing at $873 \mathrm{~K}, 1 \mathrm{~h}$ and reducing at $773 \mathrm{~K}$, with different holding times.

$80 \%$ over the ultraviolet-visible-infrared light wavelength range, and was comparable to the light absorption efficiency observed for the iron substrate with a thick porous layer and fine porous structure formed by oxidization at $1073 \mathrm{~K}$ for $1 \mathrm{~h}$ and reduction at $773 \mathrm{~K}$ for $1 \mathrm{~h}$. This result indicated that the preparation of a thin but finely porous surface layer was adequate in improving the light absorption of iron substrates up to $75-80 \%$ across the ultraviolet-visible-infrared wavelength range.

However, unlike those oxidized at $1073 \mathrm{~K}$ before reduction, the iron substrates oxidized at $873 \mathrm{~K}$ and reduced for long times exhibited high light absorption efficiency, even though pore size increased as reduction holding time increased. Further investigation is required to clarify the correlation between enhancement of light absorption efficiency of the metal substrates and the formation of porous structures on their surfaces by oxidization-reduction treatment.
The above results indicate that high light absorption efficiencies can be obtained from iron substrates with porous layers formed by oxidization-reduction treatment. Therefore, oxidization-reduction treatment could be a simple and convenient method to prepare porous asperity structures on metal surfaces, and to enhance light absorption efficiencies of metal over a wide wavelength range including infrared light, where the energy of the absorbed light could then be exploited as thermal energy.

\section{Conclusions}

Ultraviolet-visible-infrared light absorption was examined for copper and iron substrates with porous surface layers obtained by oxidizing and then reducing the substrates surfaces. It was found that pore size and porous layer thickness were dependent on the oxidization and reduction process temperatures and times. For example, a fine porous structure was obtained on the surface of oxidized iron substrate by reducing it at a low temperature. As reduction holding time was increased, the pore structure on both copper and iron substrates coarsened and pore sizes increased.

Light absorption measurements for the copper and iron substrates revealed that those with the porous layers exhibited higher light absorption efficiencies than those with mirrorpolished surfaces over a wide range of wavelengths. Higher light absorption efficiencies were obtained for metal substrates with fine porous structures. In particular, light absorption of up to $75-80 \%$ in the ultraviolet-visible-infrared wavelength range were obtained for iron substrate with a fine porous structure, prepared by oxidizing and then reducing the substrate at a low temperature. This light absorption index is much higher than light absorption by conventional metal substrates with flat surface, and comparable to gray-coloured oxide substrates such as iron or copper oxides. Since reduced metal surface generally has higher heat conductivity, metal 
substrates with porous structure on surface are expected to be beneficial materials for highly efficient use of sunlight energy. Therefore, oxidization-reduction treatment has been found to be a simple and convenient way to make porous asperity structures on the surface of metals, and to obtain high light absorption efficiencies over ultraviolet-visible-infrared light wavelengths.

\section{Acknowledgements}

This study was supported by Priority Assistance for the Formation of Worldwide Renowned Centers of Research The Global COE Program (Project: Center of Excellence for Advanced Structural and Functional Materials Design) from the Ministry of Education, Culture, Sports, Science and Technology (MEXT), Japan. We thank them most warmly for their assistance in this study.

\section{REFERENCES}

1) A. W. Smith and A. Rohatgi: Sol. Energy Mater. Sol. Cells 29 (1993) 37-49.

2) A. W. Smith and A. Rohatgi: Sol. Energy Mater. Sol. Cells 29 (1993) $51-65$.

3) S. Kajita, W. Sakaguchi, N. Ohno, N. Yoshida and T. Saeki: Nucl. Fusion 49 (2009) 095005.

4) D. H. St. John and P. C. Hayes: Metall. Mater. Trans. B 13 (1982) 117124.

5) D. H. St. John, S. P. Matthew and P. C. Hayes: Metall. Mater. Trans. B 15 (1984) 701-708.

6) S. P. Matthew, T. R. Cho and P. C. Hayes: Metall. Mater. Trans. B 21 (1990) 733-741.

7) N. Takahira, T. Tanaka, S. Hara and J. Lee: Mater. Trans. 46 (2005) 3008-3014.

8) N. Takahira and T. Tanaka: Mater. Trans. 48 (2007) 2179-2185.

9) N. Takahira, T. Yoshikawa, T. Tanaka and L. Holappa: Mater. Trans. 48 (2007) 3126-3131.

10) F. Grum and G. W. Luckey: Appl. Opt. 7 (1968) 2289-2294. 